\title{
Combining ACL Oblique Coronal MRI with the Routine Knee MRI Protocol in the Diagnosis of ACL Bundle Lesions. Can It Add A Value?
}

\author{
YASSER I. ABD ELKHALEK, M.D.*; MOUSTAFA ABDEL KAWI, M.D.** and MERHAN NASR, M.Sc.* \\ The Department of Radiodiagnosis, Faculty of Medicine, Ain Shams* and Helwan** Universities, Cairo, Egypt
}

\begin{abstract}
Background: The Anterior Cruciate Ligament (ACL) is formed of two bundles. Routine MRI protocol is usually insufficient to delineate both bundles structure, so diagnosis of AM and PL bundle injury is often limited, and development of further imaging orientation and pulse sequences is key for further improving characterization of these bundles. Accurately grading the ACL tears and differentiating between complete and partial tears as well as assessing the two bundles is key in improving the clinical and surgical management.
\end{abstract}

Aim of Study: The purpose of this study is to evaluate the diagnostic value of combining oblique coronal images to the routine MRI protocol for better assessment of the ACL bundles, thus improving management outcome by performing two MRI protocols and comparing them to the arthroscopic findings.

Patients and Methods: This prospective study was conducted between January 2016 and February 2018 in Ain Shams University Hospitals and private centers. The study population has consisted of 32 patients ( 26 men \& 6 women), ranging between 19-45 years, with mean age of 27.11 years. All patients were suspected of ACL injury referred from the outpatient clinic of orthopedic.

All patients were examined by MRI examinations composed of sagittal T2-weighted Fast-Spin Echo (FSE), coronal T2-weighted FSE, and axial T2-weighted. The sagittal images were planned on axial localizer by 15 -degree medial angling on perpendicular line to the bicondylar line and by oblique coronal MRI were the oblique coronal STIR images named were planned on midsagittal localizer in the plane parallel to the ACL and the roof of the intercondylar notch (Blumensaat's line).

A consultant orthopedic surgeon did a diagnostic arthroscopy for all included patients using standard parapatellar portals visualizing the ACL with direct visualizing of both bundles and accordingly classifying each bundle as torn or intact.

Results: The Oblique coronal MRI showed a better agreement with the diagnostic arthroscopy than routine MRI [weighted Kappa; k=0.865 \& 0.753 respectively]. For diagnosis of

Correspondence to: Dr. Yasser I. Abd Elkhalek, The Department of Radiodiagnosis, Faculty of Medicine, Ain Shams University, Cairo, Egypt
Anteromedial Bundle (AMB) tear the sensitivity was $90.9 \%$, specificity $80 \%$, with overall accuracy of $87.5 \%$. For diagnosis of Posterolateral Bundle (PLB) tear. The sensitivity was $95.5 \%$, specificity $90 \%$, with overall accuracy of $93.8 \%$.

Conclusion: The adding of oblique coronal imaging to routine MRI protocol revealed better delineation of the ACL bundles, increasing the accuracy of diagnosis each or both bundle lesions, providing significant benefit in further management and outcome in patient with suspected ACL injury.

Key Words: Magnetic resonance imaging - ACL tears Coronal oblique cuts.

\section{Introduction}

THE Anterior Cruciate Ligament (ACL) is formed of two bundles on anatomical and functional basis. The Anteromedial (AM) and Posterolateral (PL) bundles named after their insertion into the tibia they play an important role in stabilizing knee joint during its translation and rotation movements [1] The AM bundle primarily controls the anterior movement of the tibia underneath the femur, while the PL bundle primarily maintains the rotational stability of knee joint [2].

Because of its role as a key structure of the knee and the usual involvement of ligamentous injury in traumatic knee especially with sports, the

\footnotetext{
List of Abbreviation:

ACL : Anterior Cruciate Ligament.

FSE : Fast-Spin Echo.

STIR : Short-Tau Inversion Recovery.

AMB : Anteromedial Bundle.

PLB : Posterolateral Bundle.

MRI : Magnetic Resonance Imaging.

PPV : Positive Predictive Value.

NPV : Negative Predictive Value.

LR+ : Positive Likelihood Ratio.

LR- : Negative Likelihood Ratio.

BAK : Bias-Adjusted Kappa.

PABAK : Prevalence-Adjusted Bias-Adjusted Kappa.
} 
diagnosis of ACL injuries has an important part in management of such injuries. Magnetic resonance is now the gold standard in imaging the ACL associated injuries due its excellent contrast in soft tissue structures [3].

Accurately grading the ACL tears and differentiating between complete and partial tears as well as assessing the two bundles is key in improving the clinical and surgical management as not all partial tears require surgical intervention and can be managed conservatively, also the isolated single bundle tear can be managed by single bundle reconstruction surgery other than full ACL reconstruction [4].

Routine MRI protocol is usually insufficient to delineate both bundles structure, so diagnosis of AM and PL bundle injury is often limited, and development of further imaging orientation and pulse sequences is key for further improving characterization of these bundles [5] .

Aim of the work:

The purpose of this study is to evaluate the diagnostic value of combining oblique coronal images to the routine MRI protocol for better assessment of the ACL bundles, thus improving management outcome by performing two MRI protocols and comparing them to the arthroscopic findings.

\section{Patients and Methods}

This prospective study was conducted between January 2016 and February 2018 in Ain Shams University Hospitals and private centers. The study population has consisted of 32 patients (26 men $\& 6$ women), ranging between 19-45 years, with mean age of 27.11 years. All patients were suspected of ACL injury referred from the outpatient clinic of orthopedic in some hospitals.

Inclusion criteria: Skeletally mature patients, with no signs of knee osteoarthritis having positive clinical signs and symptoms, suggestive of ACL injury.

Exclusion criteria: Patients over 45 years old, previous knee surgery or endoscopy; ACL mucoid degeneration or intrasubstance ganglion cyst.

\section{MRI parameters:}

The MRI examinations were performed by using Philips ${ }^{\circledR}$ Achieva 1.5 T with phased array knee coil. The knee was positioned in extended position within the knee coil in a supine position.

\section{Routine protocol:}

The routine protocol named (protocol A) were composed of sagittal T2-weighted Fast-Spin Echo (FSE), coronal T2-weighted FSE, and axial T2weighted, having acquisition parameters as described in (Table 1). The sagittal images were planned on axial localizer by 15 degree medial angling on perpendicular line to the bicondylar line.

Table (1): MRI protocol parameters.

\begin{tabular}{|c|c|c|c|c|c|c|}
\hline & $\mathrm{TE}$ & TR & FOV & \multicolumn{2}{|c|}{ SL Gap } & Matrix \\
\hline \multicolumn{7}{|l|}{$\begin{array}{l}\text { Routine protocol } \\
\text { [protocol A]: }\end{array}$} \\
\hline \multicolumn{7}{|l|}{ - Sagittal: } \\
\hline $\mathrm{T} 2$ & 100 & 4200 & $160 \times 160$ & 3.5 & 0.5 & $256 \times 150$ \\
\hline PD & 30 & 5000 & $170 \times 170$ & 4.5 & 1.2 & $256 \times 150$ \\
\hline $\mathrm{T} 1$ & 840 & 26 & $170 \times 170$ & 3.5 & 0.3 & $256 \times 150$ \\
\hline \multicolumn{7}{|l|}{ - Coronal: } \\
\hline $\mathrm{T} 2$ fat sat & 20 & 487 & $170 \times 170$ & 5 & 1.5 & $256 \times 150$ \\
\hline \multicolumn{7}{|l|}{ - Axial: } \\
\hline $\mathrm{T} 2$ & 100 & 3.6 & $160 \times 160$ & 5.5 & 1.3 & $256 \times 150$ \\
\hline \multicolumn{7}{|l|}{$\begin{array}{l}\text { Additional protocol } \\
\text { [protocol B]: }\end{array}$} \\
\hline Oblique coronal T2 & 95 & 3500 & $150 \times 150$ & 4 & 1 & $256 \times 150$ \\
\hline
\end{tabular}

Oblique coronal MRI protocol:

The oblique coronal STIR Images named (protocol B) were planned on midsagittal localizer in the plane parallel to the ACL and the roof of the intercondylar notch (Blumensaat's line) Fig. (1) having acquisition parameters as described in (Table 1). The AM and PL bundles of the ACL are clearly delineated in the oblique coronal images Fig. (2) without requiring any knee re-positioning.

\section{MRI analysis:}

A consultant musculoskeletal radiologist firstly reviewed the routine imaging exam (protocol A) and categorized the ACL as intact, partial-thickness tear or full-thickness tear. Then he reviewed the oblique coronal images (protocol B) and categorized the ACL as intact Fig. (2), complete tear of both bundles Fig. (3A,B), AM bundle tear Fig. $(4 \mathrm{~A}-\mathrm{C})$ or PL bundle tear Fig. (5A-C).

\section{Knee arthroscopy:}

A consultant orthopedic surgeon did the diagnostic arthroscopy for all included patients using standard parapatellar portals visualizing the ACL with direct visualizing of both bundles and accordingly classifying each bundle as torn or intact. The torn bundle was identified as the bundle with lost continuity of its fibers as well loss of its tautness by direct probing. 


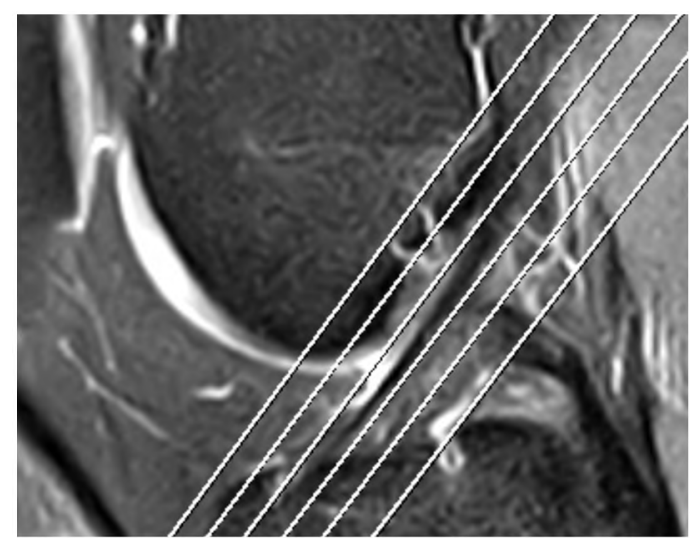

Fig. (1): Yellow lines parallel to the ACL longitudinal axis, in the mid sagittal localizer image (image from one of this study patients).

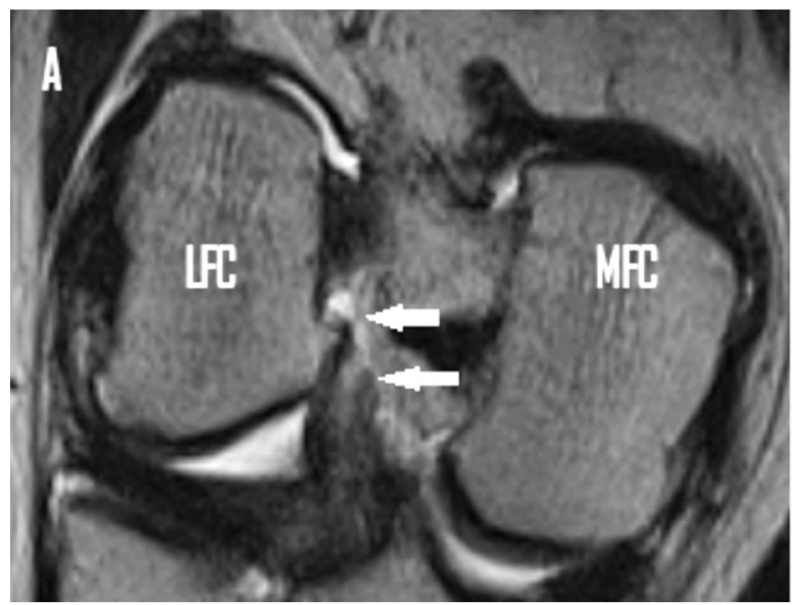

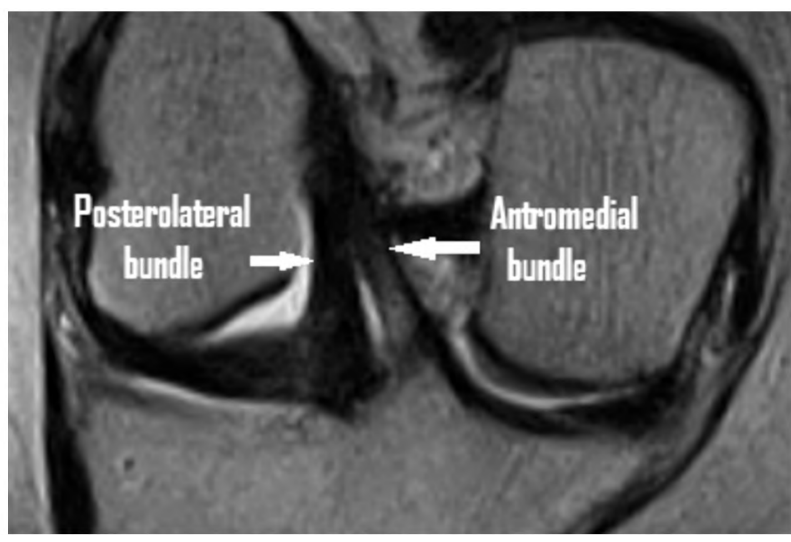

Fig. (2): Coronal oblique T2-weighted image of the ACL revealing normal posterolateral and anteromedial bundles ACL with a thin bright linear band of high signal between them. (images from one of this study patients).

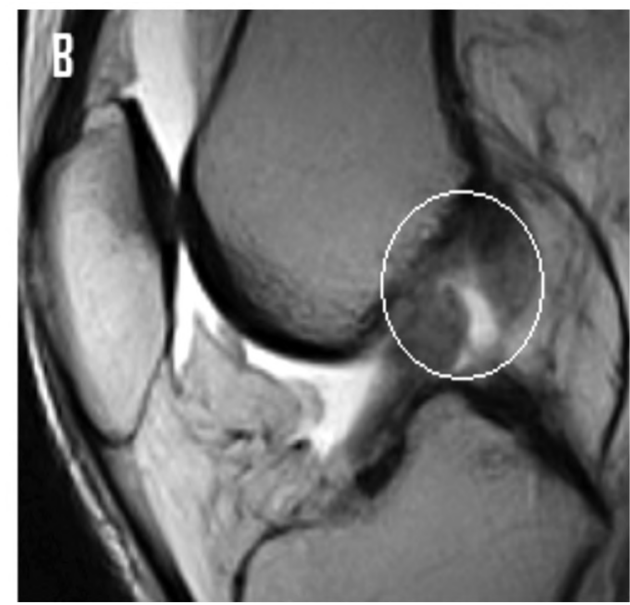

Fig. (3): A 37 years old male patient from our study complaining of knee pain after trauma and giving a way (A) Coronal oblique T2 WI revealed torn both AM and PL bundles [white arrows] clearly differentiated from each other with regional fluid signal related to the femoral attachment to the lateral femoral condyle. (B) Corresponding mid sagittal T2 WIs revealed proximal femoral full thickness tear [circle].
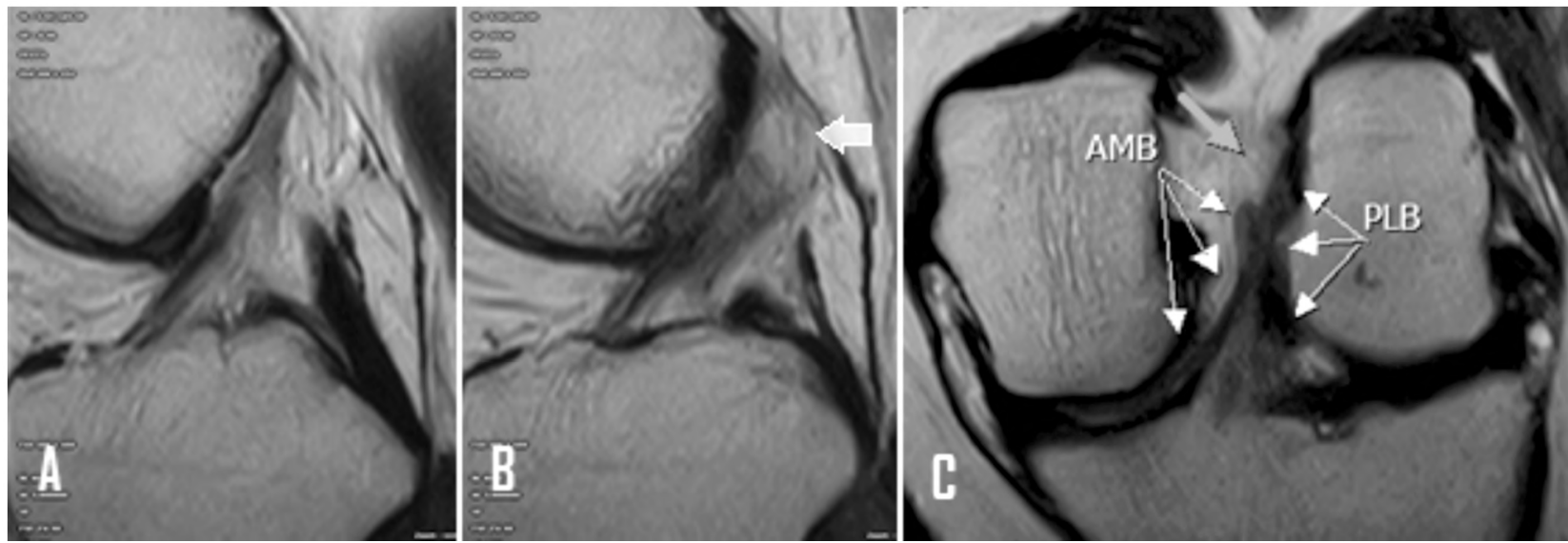

Fig. (4): A 37 years old male patient from our study complaining of knee pain after trauma and giving a way (A, B) Midsagittal two consecutive images showing mild haziness and thickening of the posterior margins of the ACL with intermediate signal opposite to its femoral attachment. (C) Corresponding oblique coronal image revealed avulsion tear of the proximal femoral attachment of the anteromedial bundle [orange arrow]. The posterolateral bundle appears intact. 

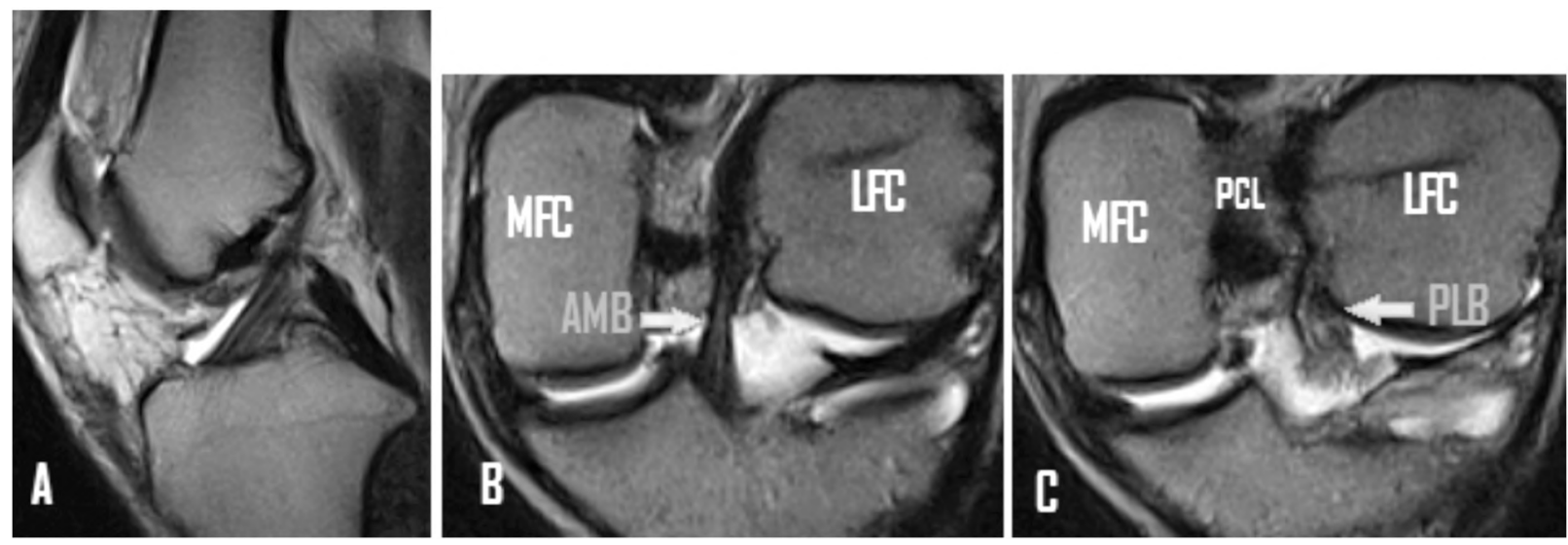

Fig. (5): A 31 years old male patient from our study complaining of knee pain after trauma (A) Mid sagittal T2 image with apparently intact ACL showing minimal linear bands among its fascicles and mild haziness related to its posterior margins. (B,C) Two consecutive oblique coronal T2-images showing intact anteromedial bundle and avulsed distal posterolateral bundle from its tibial attachment with frayed distal end and slightly retracted/corrugated proximal fibers. Note a displaced inner meniscal fragment in conjunction with a displaced medial meniscus bucket handle tear. AMB $=$ Anteromedial Bundle, PLB $=$ Posterolateral Bundle, MFC $=$ Medial Femoral Condyle and LFC $=$ Lateral Femoral Condyle.

\section{Statistical analysis:}

We used the following terms to represent diagnostic accuracy: Sensitivity, specificity, Positive Predictive Value (PPV), Negative Predictive Value (NPV), positive likelihood ratio ( $\mathrm{LR}+)$, negative likelihood ratio (LR-) and overall accuracy. Statistical analysis was done, using MedCalc $\odot$ version 15.8 (MedCalc@ Software bvba, Ostend, Belgium). Inter-method agreement concerning the assessment of binary outcomes was evaluated by calculating Cohen's kappa (ic), Bias-Adjusted Kappa (BAK) and Prevalence-Adjusted Bias-Adjusted Kappa (PABAK) coefficients. Weighted is was used to examine the inter-method agreement for assessing outcomes rated on an ordinal scale. The accuracy of protocol B for a single bundle tear was compared with diagnostic arthroscopy, as a current gold standard method. Contingency tables were used to calculate the sensitivity, specificity, PPV and NPV; true-and false-positive rates; and correct classification (accuracy) and misclassification rates.

\section{Results}

For the diagnosis of an AMB tear (Table 2), the oblique coronal protocol had $90.9 \%$ sensitivity, $80.0 \%$ specificity, $87.5 \%$ accuracy, $20 \%$ falsenegative rate, $20 \%$ false-positive rate, $90.9 \% \mathrm{PPV}$ \& $80 \%$ NPV. Positive likelihood ratio [LR+] 4.55 and negative likelihood ratio [LR-] 0.11, A strong inter-method agreement was achieved [K-value= $.71, \mathrm{BAK}=.71, \& \mathrm{PABAK}=.75]$.

Among the 32 patient there were 20 tears that accurately diagnosed by coronal oblique images and confirmed by arthroscopy (true positive) and 8 cases were negative by both the arthroscopy and MRI (true negative). 2 cases were diagnosed as partial thickness bundle tear by oblique coronal MRI were negative by arthroscopy (false positive). Another 2 cases were diagnosed as intact and we found partial thickness tear by arthroscopy (false negative) (accuracy 87.5\%).

For diagnosing a PLB tear (Table 3), the oblique coronal protocol had a $95.5 \%$ sensitivity, $90 \%$ specificity, $93.8 \%$ accuracy, $10 \%$ false-positive rate, $10 \%$ false-negative rate, $95.5 \% \mathrm{PPV}, 90 \%$ NPV. Positive likelihood ratio [LR+] 9.55 and negative likelihood ratio [LR-] 0.05 . A very strong inter-method agreement was achieved $[\mathrm{ic}=.85$, $\mathrm{BAK}=.85, \& \mathrm{PABAK}=.88]$.

Among the 32 patient there were 21 tears that accurately diagnosed by coronal oblique images and confirmed by arthroscopy (true positive) and 9 cases were negative by both the arthroscopy and MRI (true negative). 1 case was diagnosed as partial thickness bundle tear by oblique coronal MRI were negative by arthroscopy (false positive). Another case was diagnosed as intact and we found partial thickness tear by arthroscopy (false negative) (accuracy 93.8\%).

The oblique coronal protocol showed higher inter-method agreement with the arthroscopy $(\mathrm{x}=0.865,95 \%$ CI $0.718-1.000$; very strong agreement) than the routine protocol did (ic $=0.753,95 \%$ CI 0.586-0.920; strong agreement). 
Table (2): Diagnostic performance of oblique coronal protocol for diagnosis of Anteromedial Bundle (AMB) tear.

\begin{tabular}{ll}
\hline Statistic & Value \\
\hline Sensitivity & $90.9 \%$ \\
Specificity & $80.0 \%$ \\
False positive rate & $20.0 \%$ \\
False negative rate & $20 \%$ \\
Prevalence & $68.8 \%$ \\
Positive predictive value (PPV) & $90.9 \%$ \\
Negative predictive value (NPV) & $80.0 \%$ \\
Positive likelihood ratio (LR+) & 4.55 \\
Negative likelihood ratio (LR-) & 0.11 \\
\hline
\end{tabular}

Table (3): Diagnostic performance of oblique coronal protocol for diagnosis of Posterolateral Bundle (PLB) tear.

\begin{tabular}{ll}
\hline Statistic & Value \\
\hline Sensitivity & $95.5 \%$ \\
Specificity & $90 \%$ \\
False positive rate & $10 \%$ \\
False negative rate & $10 \%$ \\
Prevalence & $68.8 \%$ \\
Positive predictive value (PPV) & $95.5 \%$ \\
Negative predictive value (NPV) & $90.0 \%$ \\
Positive likelihood ratio (LR+) & 9.55 \\
Negative likelihood ratio (LR-) & 0.05 \\
\hline
\end{tabular}

\section{Discussion}

Many anatomical and bio-mechanical studies had described the significant contributions of both $\mathrm{AM}$ and PL bundles in knee function and proved that no single bundle alone can reproduce the biomechanical function of fully intact ACL [6] .

The AMB mainly prevents the anterior translation of tibia in relation to the femur. The PLB functions as a keeper of the rotational knee movements in case of pivoting, twisting or jumping $[7,8]$ AMB also strengthen and tighten the knee on flexion while PLB shortens it [9].

Routine coronal and sagittal sequences can allow accurate detection of the AMB while the PLB can't be well visualized secondary to the blurring from partial volume effect of the lowresolution images of the bundles in sequential images and they would appear as one bundle [10]

A high field 3T superconductive MRI can well delineate the two bundles in different views as found by Steckel et al., [11] in his study on cadaveric knees.

A study done by Lawrence et al., [12] showed that MRI accurately diagnosed only one of nine partial ACL tears confirmed by arthroscopy [12] However in another study done by Chang et al., [13] showed overall diagnostic accuracy of MRI in partial tears of $83 \%$ [13].
As the MRI nowadays is the modality of choice in imaging of ACL injuries, the need for providing and producing additional techniques by adding parallel slicing along the longitudinal ACL axis, added to the routine MRI protocol, thus providing remarkable role in ACL tears, especially those involving just one bundle as they have traditionally been difficult to diagnose [14].

The accurate comment on different ACL bundle tears, gives more information and precise location of the tear providing a more prognostic value in management of these lesions, as the newly developed single specific bundle reconstruction approaches was surgically introduced, limiting the commonly used complete ACL reconstruction surgery [15]. In addition, some of the patients with single bundle tear may be managed conservatively, so, good differentiation of ACL single bundle tear is of great concern to the orthopedic surgeon $[16,20]$

Kamal et al., [17] performed a study using oblique axial MR protocol, aiming to increase accuracy of detecting single bundle tear. Their results showed increased sensitivity up to $88 \%$ for antromedial bundle tear and up to $89 \%$ for posterolateral bundle tear [17].

Another study performed by Volokhina et al., [7] evaluating the value of "gap" and "foot print" signs in regular MRI to diagnose the posterolateral bundle tears. Gap and footprint signs are suggestive of posterolateral bundle tear of the ACL, and the presence of 1 or both imaging findings should alert the radiologist to the possibility of a posterolateral bundle tear. The "gap" sign, referring to the gap between the medial aspect of the lateral femoral condyle and the lateral aspect of the proximal Anterior Cruciate Ligament (ACL) attachment. "footprint" sign, referring to the incomplete coverage of the lateral aspect of the interspinous area of the tibia by the distal Anterior Cruciate Ligament (ACL). The gap sign reveals $72 \%$ sensitivity and $68 \%$ specificity in coronal plane, $52 \%$ sensitivity and $53 \%$ specificity in axial plane. The foot print sign reveals $75 \%$ sensitivity and $80 \%$ specificity in coronal plane. The existence of one sign or both had $82 \%$ sensitivity and $58 \%$ specificity [7].

In our study, we evaluated the diagnostic value of using oblique coronal MRI technique for diagnosing ACL single bundle injury and adding it to the routine MRI protocol with a 1.5 Tesla MRI scanner, which does not add any significant time or any additional cost to the exam, as well as, reduce the need of performing routine MRI examination on the less available and relatively more expensive 3 -Tesla scanner. 
Among the 32 patient there were 20 AMB tears that accurately diagnosed by coronal oblique images and confirmed by arthroscopy (true positive) and 8 cases were negative by both the arthroscopy and MRI (true negative). 2 cases were diagnosed as partial thickness bundle tear by oblique coronal MRI were negative by arthroscopy (false positive). Another 2 cases were diagnosed as intact and we found partial thickness tear by arthroscopy (false negative). And so, the oblique coronal protocol had $90.9 \%$ sensitivity, $80.0 \%$ specificity, $87.5 \%$ accuracy, $9.1 \%$ false-negative rate, $20 \%$ falsepositive rate, $90.9 \%$ PPV \& $80 \%$ NPV. Positive likelihood ratio [LR+] 4.55 and negative likelihood ratio [LR-] 0.11 .

As regard the PLB there were 21 tears that accurately diagnosed by coronal oblique images and confirmed by arthroscopy (true positive) and 9 cases were negative by both the arthroscopy and MRI (true negative). 1 case was diagnosed as partial thickness bundle tear by oblique coronal MRI were negative by arthroscopy (false positive). Another case was diagnosed as intact and we found partial thickness tear by arthroscopy (false negative) and so, the oblique coronal protocol had a $95.5 \%$ sensitivity, 90\% specificity, $93.8 \%$ accuracy, 10 $\%$ false-positive rate, $4.5 \%$ false-negative rate, 95.5\% PPV, 90\% NPV. Positive likelihood ratio [LR+] 9.55 and negative likelihood ratio [LR-] 0.05 .

The study clearly declares the superiority of the oblique coronal technique in delineating the ACL two bundles adding more data regarding ACL bundle injuries. Our results were matched with Staeubli et al., [18] results that the parallel oblique coronal images were method of choice in differentiating diagonal anatomical course of the ACL bundle tears. Moreover, oblique coronal images give clear visualization and estimation of changes in signal and width of the ACL bundles comparing to the routine protocol [18].

Roychowdhury et al., [19] used conventional axial and coronal sequences in the diagnosis of partial tears of the ACL and they were able to discriminate stable tears from unstable tears either partial or incomplete of sensitivity $100 \%$ and specificity $96 \%$ [19].

Our results also match the results by Hong et al., [5] which demonstrated that the diagnostic accuracy for ACL tears was improved with additional oblique coronal planes compared with the standard orientations.
Our study was limited by refusal of some patient with positive MRI findings of ACL tear to perform diagnostic arthroscopy excluding them from the study. Another limitation is that only one radiologist interprets the images that could be a bias. We also recommend performing new study with $3 \mathrm{~T}$ MRI machine for more accurate results although 1.5T was enough in diagnosis for our cases.

\section{Conclusion:}

Adding oblique coronal thin slices to the routine MRI protocol provides a better delineation of ACL bundles, improving the diagnostic accuracy of detecting single/double bundle injury, without adding additional time or cost, hence improving the management of these injuries.

\section{References}

1- YAMAMOTO Y., HSU W.H., WOO S.L., VAN SCYOC A.H., TAKAKURA Y. and DEBSKI R.E.: Knee stability and graft function after anterior cruciate ligament reconstruction: A comparison of a lateral and an anatomical femoral tunnel placement. Am. J. Sports Med., 32: 182532, 2004.

2- ZANTOP T., HERBORT M., RASCHKE M.J., FU F.H. and PETERSEN W.: The role of the anteromedial and posterolateral bundles of the anterior cruciate ligament in anterior tibial translation and internal rotation. Am. J. Sports Med., 35: 223-7, 2007.

3- DUTHON V.B., BAREA C., ABRASSART S., FASEL J.H., FRITSCH D. and MÉNÉTREY J.: Anatomy of the anterior cruciate ligament, Knee Surgery, Sports Traumatology, Arthroscopy,March, Volume 14, Issue 3, 204-13, 2006.

4- CHEN W., XIE B., GUO-HONG Z., LARS G., LI-WEN T., WANG J., ZHANG S., YA-MING W., ZHANG Y. and CHENO L.: Oblique coronal view of the ACL doublebundle: Comparison of the Chinese Visible Human dataset and low-field mri, experimental and therapeutic medicine; 6: 606-10, 2013.

5- HONG S.H., CHOI J.Y., LEE G.K., CHOI J.A., CHUNG H.W. and KANG H.S.: Grading of anterior cruciate ligament injury. Diagnostic efficacy of oblique coronal magnetic resonance imaging of the knee. J. Comput. Assist. Tomogr., 27: 814-9, 2003.

6- KATO Y., HOSHINO Y., INGHAM J.M. and FREDDIE H.F.: Anatomic double-bundle anterior cruciate ligament reconstruction. J. Orthop. Sci., 15: 269-76, 2010.

7- VOLOKHINA Y.V., SYED H.M., PHAM P.H. and BLACKBURN A.K.: Two Helpful MRI Signs for Evaluation of Posterolateral Bundle Tears of the Anterior Cruciate Ligament. The Orthopaedic Journal of Sports Medicine, 3 (8), 2015.

8- CHAMBAT P., GUIER C., SONNERY-COTTET B., FAYARD J.M. and THAUNAT M.: The evolution of ACL reconstruction over the last fifty years. Int. Orthop., 37: 181-6, 2013. 
9- HOLLIS J.M., TAKAI S., ADAMS D.J., HORIBE S. and WOO S.L.: The effects of knee motion and external loading on the length of the Anterior Cruciate Ligament (ACL): A kinematic study. J. Biomech. Eng., 113: 208 $14,1991$.

10- STARMAN J.S., VANBEEK C., ARMFIELD D.R., et al.: Assessment of normal ACL double bundle anatomy in standard viewing planes by magnetic resonance imaging. Knee Surg. Sports Traumatol. Arthrosc., 15: 493-9, 2007.

11- STECKEL H., VADALA G., DAVIS D., MUSAHL V. and FU F.H.: 3-T MR imaging of partial ACL tears: A cadaver study. Knee Surg. Sports Traumatol. Arthrosc., 15: 1066-71, 2007.

12- LAWRANCE J.A., OSTLERE S.J. and DODD C.A.: MRI diagnosis of partial tears of the anterior cruciate ligament. Injury, 27: 153-5, 1996.

13- CHANG M.J., CHANG C.B., CHOI J.Y., WON H.H. and KIM T.K.: How useful is MRI in diagnosing isolated bundle ACL injuries? Clin. Orthop. Relat. Res., 471: 3283-90, 2013.

14- PETERSEN W. and ZANTOP T.: Partial rupture of the anterior cruciate ligament. Arthroscopy, 22 (11): 1143-5, 2006.

15-LORENZ S. and IMHOFF A.B.: Reconstruction of partial anterior cruciate ligament tears. Oper. Orthop. Traumatol., 26 (1): 56-62, 2014.

16- SIEBOLD R. and FU F.H.: Assessment and augmentation of symptomatic anteromedial or posterolateral bundle tears of the anterior cruciate ligament. Arthroscopy, 24: 1289-98, 2008.

17- KAMAL H.A., ABDELWAHAB N. and EL-LIETHY N.E.: The role of oblique axial MR imaging in the diagnosis of ACL bundle lesions. The Egyptian Journal of Radiology and Nuclear Medicine, 46: 683-93, 2015.

18-STÄUBLI H.U. and RAUSCHNING W.: Tibial attachment area of the anterior cruciate ligament in the extended knee position. Anatomy and cryosections in vitro complemented by magnetic resonance arthrography in vivo. Knee Surg. Sports Traumatol. Arthrosc., 2: 138-46, 1994.

19- ROYCHOWDHURY S., FITZGERALD S.W., SONIN A.H., PEDUTO A.J., MILLER F.H. and HOFF F.L.: Using MR imaging to diagnose partial tears of the anterior cruciate ligament: Value of axial images. A.J.R. Am. J. Roentgenol., 168: 1487-91, 1997.

20- XU B., ZHANG H., LI B. and WANG W.: Comparison of magnetic resonance imaging for patients with acute and chronic anterior cruciate ligament tears. Medicine (Baltimore), 97 (10), 2018

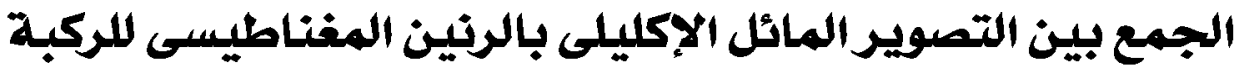

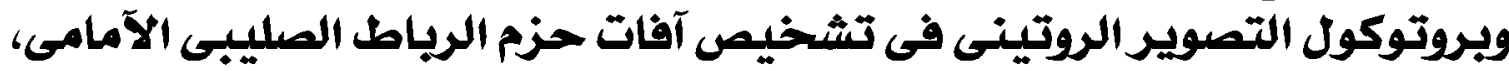

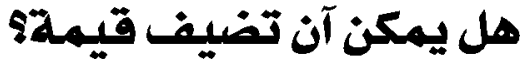

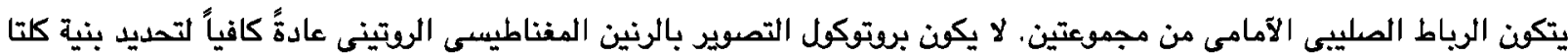

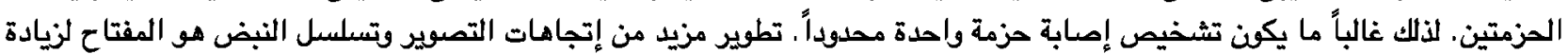

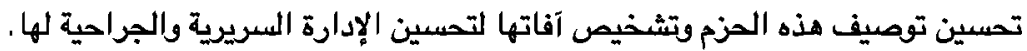

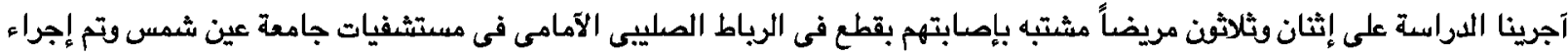

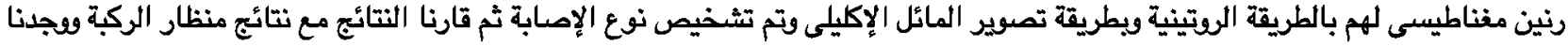
دقة عالية فى تشخيص نوع الإصابة لكل من الحزمتين لالرياط الصليبي الآمامى.

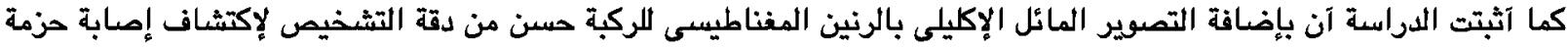
مفردة/مزدوجة، دون إضافة وقت آو تكلفة إضافية، وبالتالى تصسين إدارة الإنى هذه الإصنابات. 